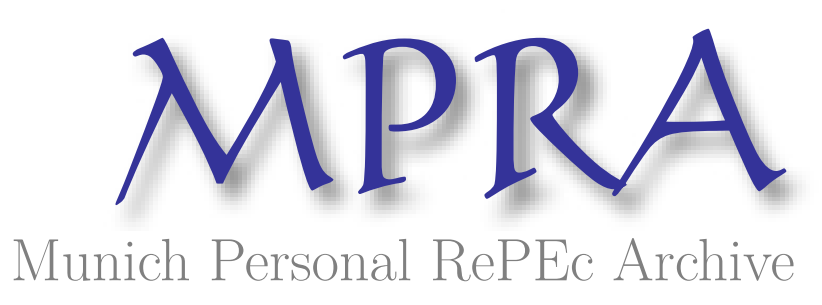

\title{
Spatial Allocation of Economy as a Fiber Bundle
}

Van-Ilya, Andrei

22 August 2012

Online at https://mpra.ub.uni-muenchen.de/40820/

MPRA Paper No. 40820, posted 23 Aug 2012 10:15 UTC 


\title{
Spatial Allocation of Economy as a Fiber Bundle
}

\author{
Andrei Van-Ilya
}

August 2012

\begin{abstract}
This paper considers the approach to specification and modeling of transport influence on spatial allocation of economy, which is essentially new for economics. By applying the concept of fiber bundle, a general model of spatial allocation of market with regard to transport costs is developed. Corresponding mathematical formulation of model equilibrium condition and transition dynamics is stated based on the principle of least action and gauge invariance. Further development of obtained theoretical results within the framework is reviewed.
\end{abstract}

Keywords: transport costs, market allocation in space, fiber bundle, gauge model

JEL Classification: C69, R12, R49 


\section{Introduction}

This paper considers the approach to specification and modeling of transport influence on spatial allocation of economy.

The study of spatial allocation of economy and its determinants has played a marginal role in economic theory most of the time since the emergence of political economy (Blaug, 1997; p.596). Such a role might be correct in the XIX century under the assumption of additive character of transport influence on allocation of economic activities. However, because of urbanization and introduction of multimodal transportation this assumption soon proved to be inconsistent with empirical facts. Nevertheless, lack of appropriate mathematical tools has limited research into the subject for a long time.

Recent theoretical studies of transport influence on spatial allocation of economy are carried out by simulation of either multiregional economies or agglomerations with endogenous growth and trade with economies of scale and imperfect competition in the presence of transport costs. Such models are being developed based on Dixit and Stiglitz approach to formalization of Chamberlinian monopolistic competition. The symmetric CES utility function, introduced by them, is analytically tractable. That induced a great amount of papers on different issues, which eventually formed "new economic geography" (NEG). It as well has played a key role in the emergence of endogenous growth theory (Aghion, Durlauf, 2005) and new trade theory (Neary, 2000).

NEG literature on spatial allocation of economy was pioneered by Fujita (1988), Krugman (1991) and Venables (1996). Since then the amount of articles on the subject has been growing too rapidly to be overviewed full and yet brief. In order to get a profound comprehensive survey of origins, development and results obtained in new economic geography, one may refer to the papers of Fujita and Mori (2005a), Ottaviano and Thisse (2000), Mikkelsen (2004) and Krugman (2011).

The example of detailed study of transport influence on allocation of economic activities within NEG framework is one held by Fujita and Mori (2005b). They analyzed impacts of transport costs on the spatial patterns of economic agglomeration. The main theoretical result is that in presence of several agglomeration and dispersion forces the relative level and dynamics of transport costs for different goods is the critical determinant of economic spatial patterns. Moreover, the effect of transport costs change is not monotonic.

Although NEG models succeeded in revealing and detailing the role of transport costs in spatial allocation of economy, they suffer from several serious flaws.

First, in the majority of papers on the subject transport costs take Samuelson's iceberg form, which means they are incurred in the goods shipped, and thus a fraction of a good shipped is spent on transportation. Such an 
approach might be convenient for technical reasons of modeling but obviously is not empirically grounded. That may distort the nature of conclusions.

Second, transport costs are invariant to location. It may be valid for research of any small part of the economy. However, it is too high level of generalization for research of multiregional economies and agglomerations.

Third, values and dynamics of transport costs are exogenous to economic processes. In short term it could be considered correct. However, agglomeration (dispersion) as well as transport development is a long-term process. In addition, obviously evolution of transport infrastructure is closely related to spatial patterns of economic allocation. Thereby in order to specify transport influence on spatial allocation of economy essentially, it is necessary to develop endogenous mechanism of this influence.

To sum up, recent theoretical studies within NEG framework lack unified approach to specification and modeling of transport influence (and transport costs, in particular) on spatial allocation of economy, which could integrate economic and physic attributes of the subject. In author's opinion, it restrains further research on location of economic activities. In order to go on studies and draw new theoretical conclusions, it is essential to develop such an approach.

This paper presents the approach to specification and modeling of transport costs influence on spatial allocation of economy, which is essentially new for economic theory. It is an application of the concept of fiber bundle and gauge theory. The theory of fiber bundles is currently a key section of differential geometry. Its application in gauge models plays an extraordinarily important role in modern physics (field theory). As it will be clear later, it is the best way to overcome the above-mentioned drawbacks of new economic geography models.

Unfortunately, novelty of the approach also means a great amount of notions, which are far out of economists' mathematical background. To master the approach quite sufficiently, it is necessary to have certain knowledge in differential geometry, group theory and gauge theory. Aware of one's while to get such knowledge, the author aims to ease the insight to the concept. In order to do that, in this article the use of mathematical notions, unfamiliar to economists, is reduced to the inevitable level. For the same reason, a model, developed in section 3, is quite a simple one. That is, this paper may be used to get apprehension of the approach.

For those, who would like to check the process of considerations in detail, it is worthwhile to study notions and statements, mentioned in this paper, by referring to more profound literature: i.e. Michor (1991), Neeb (2010), Husemoller (1993), Rubakov (2002).

The rest of this paper is organized as follows. Section 2 introduces the basic framework that covers the concept and methodology of the approach. In section 3 a simple mathematical model, which is the variant of applying the approach, is presented. Section 4 discusses advantages, new issues to be 
explored and further development within the framework. Section 5 contains summary of the paper.

\section{Basic framework}

The main idea of the presented approach is to "geometrize" transport and corresponding economic spatial variables in the following way. First, let denote real space, in which economy is located, as base space. Models of the latter will be a sphere for the case of world economy (or world market) and a plane for any local part of the world economy. Then for every point of this base space we may add an imaginary space of local prices and transport costs. Locally, in a small neighborhood of a point of base space such an addition is the Cartesian product of base and imaginary spaces and may look like a Euclidean three-dimensional space (two physical coordinates $\left(\mathrm{x}_{1}, \mathrm{x}_{2}\right)$ and an imaginary coordinate for economic and transport spatial variables). In fact, by this procedure of imaginary space addition we have built a fiber bundle: total space $E$, that consists of real space (base $B$ ), "transport-economy" imaginary space (one-dimensional fiber $F$ ) and continuous surjection $\pi: E \rightarrow B^{1}$.

Next, consider a common market located in space $B$. To improve clarity (but with no impact on the matter of considerations), assume a) only one type of goods is traded; b) demand curve is equal for all points of $B$. In the obvious way spatial allocation of this commodity is descript by 2 economic variables: price and quantity, which will be considered spatial variables, as well: they may be different for each point of base space $B$. Local quantities are cleared according to exogenous local demand curves and endogenous local prices and local supply. Since we are developing a model of transport influence on market spatial allocation and define the fiber as the imaginary space of transport and corresponding economic spatial variables, the price has its values in fibers in respect that it depends on transport costs and this dependence is additive. Then, spatial allocation of the common market is mapped by a complex number (local quantity; the inverse of local price), which is defined as a function of physical coordinates, and whose imaginary part (local price) could be considered the third (imaginary) coordinate of the traded commodity. We have applied the inverse instead of the local price itself for the reasons that will be clear later.

The inverse of the projection $\pi$ is called section ${ }^{2}$. If the topology of base space is trivial, one may identify it with a surface embedded in 3-dimensional

\footnotetext{
${ }^{1}$ A fiber bundle (E, p, B, F) consists of manifolds $\mathrm{E}, \mathrm{B}, \mathrm{F}$ and surjective mapping $\mathrm{p}$ : $\mathrm{E} \rightarrow \mathrm{B}$ with the following property of local triviality: each $\mathrm{x} \in \mathrm{B}$ has an open neighborhood $\mathrm{U}$, for which there exists a homeomorphism between $\mathrm{p}^{-1}(\mathrm{U})$ and the product space $\mathrm{U} \times \mathrm{F}: \psi_{U}: p^{-1}(U) \rightarrow U \times F$, in such a way that $\mathrm{p}$ carries over to the projection onto the first factor of the product space: $p=\psi_{U} \circ p_{U}$, where $p_{U}: U \times F \rightarrow U$ is the natural projection.

${ }^{2}$ A section of a fiber bundle $(\mathrm{E}, \mathrm{p}, \mathrm{B}, \mathrm{F})$ is continuous mapping s: $\mathrm{B} \rightarrow \mathrm{E}$, such that $p(s(x))=x$ for any point $x$ of base space $B$.
} 
(two physical coordinates and local price) space $E$. The existence of globally trivial sections (and hence existence of global common market) is not evident in general. The curvature of this surface is an expression of price inequality. Its topology affects allocation of the commodity. According to the presented approach, (under equilibrium trade flows) this inequality is caused by spatially variant transport costs. The diagram, that pictures the concept of "transporteconomy" fiber bundle model, is shown on Figure 1. The mechanism of interaction between local prices and transport costs in this fiber bundle model of market is as follows.

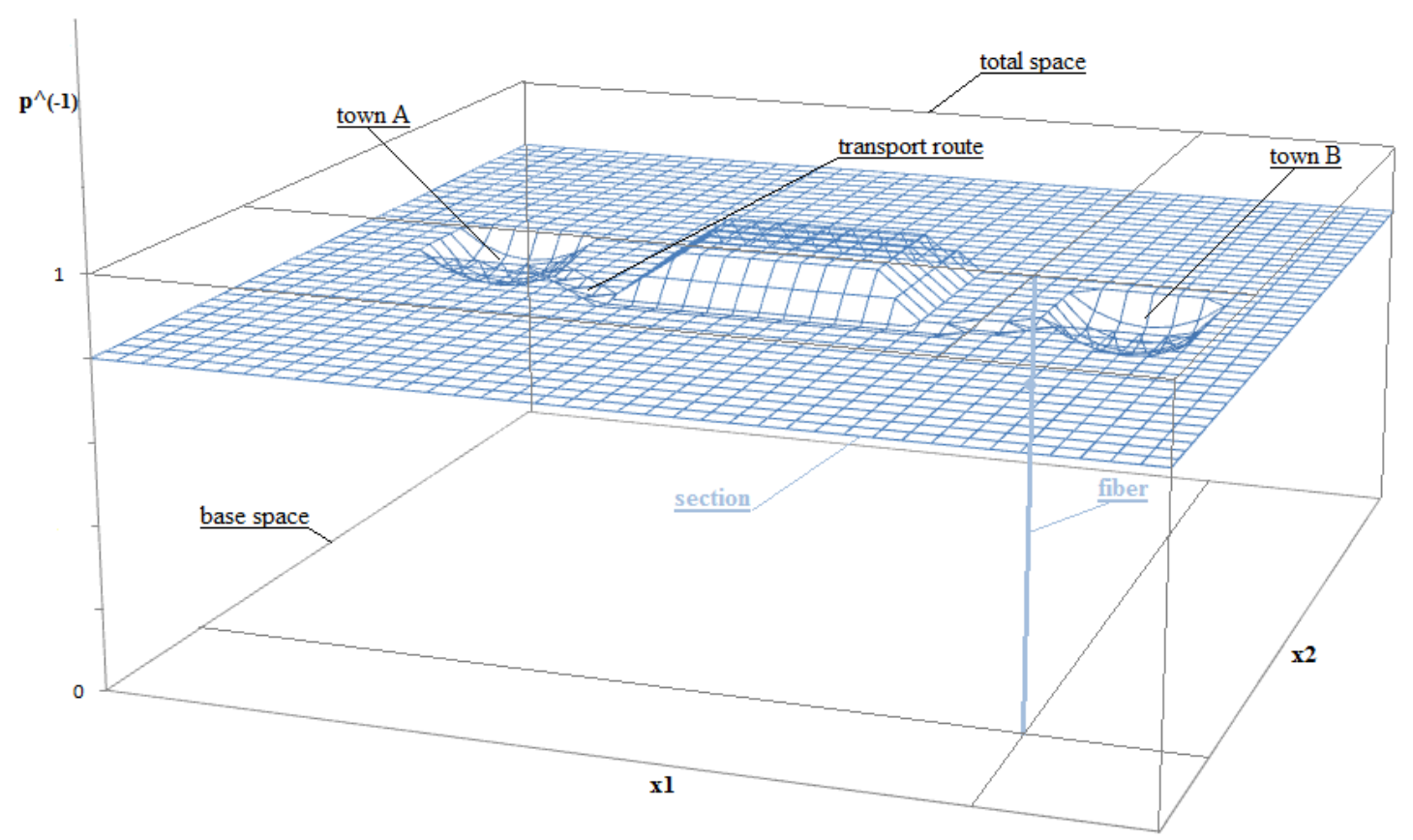

Figure 1. Fiber bundle model of the market

The equilibrium dynamics is based on the principle of least action. According to the latter among all the curves, that connect two points, the one of the minimum length corresponds to the minimum of action ${ }^{3}$. Moreover, such a curve is actually the real path of any physical system. Since we "fiber" total space by transport costs, this principle would mean minimization of transportation action. The implementation of the principle of least action is relevance to this economic model rather than formal requirement of the approach for two reasons. First, transportation is a physical action, and thus efficient spatial allocation of transport network corresponds to this principle and (as it will be clear below) is actually under its influence. Second, this principle is a sound assumption according to the microeconomics: other things being equal, it means that suppliers aim to minimize transport costs and distance while they

\footnotetext{
${ }^{3}$ To be exact, the action is stationary to first order: $\partial S=0$.
} 
choose ways to transport and places to supply the commodity. That is, suppliers maximize their profits.

Next, if transport costs are zero all over the base space $B$, prices are equal all over the base space $B$ : the equilibrium spatial allocation of the commodity will depend only on local demand and global supply. It also means that abovementioned surface becomes flat. Besides, due to the principle of least action, trade between any pair of points in $B$ will flow along the straight line.

Since we define local prices as values in fibers, positive spatial variance of transport costs would evidently cause spatial price inequality. Spatial allocation of the commodity will follow spatial price variation in the obvious way: the higher the local equilibrium price, the higher the local supply. Besides, implementation of the principle of least action requires that the equilibrium trade corresponds with the minimum of change in transport costs from one point of $B$ to another: trade flows along the projection of $E$-curve of minimum (in $E$ ) length. Basically, the latter stands for endogenous emergence of transport network.

It makes sense to bring the argument in the reverse order: deviation of commodity allocation in base space from spatially homogeneous equilibrium (local demand is considered equal all over the base space) and deviation of trade flows from straight lines are caused by deviation of local prices in fibers from the common global equilibrium price. Due to the market clearing, existence of local prices calls for existence of explanatory spatial variable in our fiber bundle model. In other words, in order to explain distortion of allocation of the commodity from the equilibrium, specified only by local demand, (and distortion of trade flows in $B$ from straight lines) endogenous requirement for positive spatially variant transport costs (transport network) appears. It is an important advantage of the constructed fiber bundle model of market.

The distinction of the curved price surface (section) and the curved line from respectively the flat and the straight ones is expressed in a notion of a connection (for the introduction to the notion see i.e. Way, 2010). Connection defines how far the third (imaginary) dimension - local price transforms from point to point of total space. Meanwhile according to the model, transport costs cause spatial variation of price. That is why; local values of transport costs are identified with the components of local connection forms ${ }^{4}$ of our "transporteconomy" fiber bundle.

\footnotetext{
${ }^{4} \mathrm{~A}$ connection form $\omega$ on a principal bundle $\mathrm{E}$ is a differential 1-form (with values in Lie algebra $\Theta$ of the group $\mathrm{G})$, that satisfies the following properties:

1) $\omega(\xi a)=a$ for every point $\xi \in E$ and $a \in \Theta$.

2) $\omega(d \xi g)=g^{-1} \omega(d \xi) g$ for $d \xi \in T_{\xi}$ and $g \in G$.
}

Where $T_{\xi}$ is a vector space of tangent vectors at point $\xi$.

A Lie algebra is a vector space $\mathrm{g}$ over some field $\mathrm{F}$ together with a binary operation $[\cdot, \cdot]: g \times g \rightarrow g$ (called the Lie bracket), which satisfies the following properties:

$$
\text { i) }[a x+b y, z]=a[x, z]+b[y, z] ; i i)[x, x]=0 ; i i i)[x,[y, z]]+[y,[z, x]]+[z,[x, y]]=0
$$




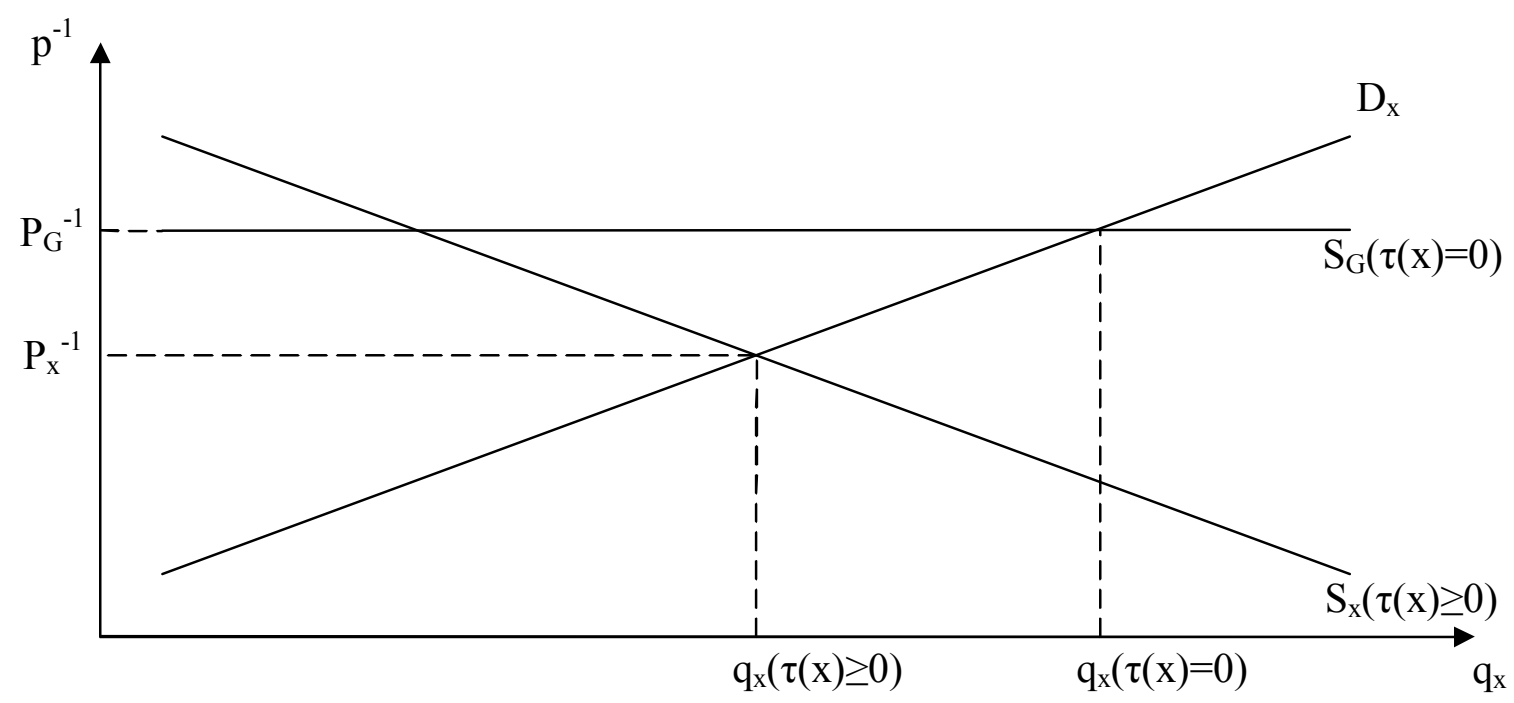

Figure 2. Equilibrium on the local market in the presence and in the absence of transport costs

Before moving on to formulate a gauge model of transport costs influence on spatial allocation of market, it is necessary to specify the type of the constructed "transport-economy" fiber bundle.

First, we may add commutative group ${ }^{5}$ structure on the set of prices in the presence of transport costs. Indeed, all the local prices in the presence of transport costs will be equal or higher than the global equilibrium price in the absence of transport costs. From Figure 2 it is clear that the inverse of the latter is the greatest element of the set of inverses of local prices. Obviously, zero is its least element. Let define half the inverse of the global equilibrium price in the absence of transport costs as the identity element. All other elements of the set can be remeasured based on this identity element. Thus, for each element in the defined group, there exists an inverse element. Closure and associativity hold true for prices a priori.

for all scalars a, b in F and all elements $\mathrm{x}, \mathrm{y}, \mathrm{z}$ of the vector space. Lie groups may define associated real Lie algebra by adding the tangent space to the identity element of the corresponding group.

${ }^{5} \mathrm{~A}$ group $\mathrm{G}$ is a set together with a binary operation (addition or multiplication), which satisfy the following (group) properties:

1) closure: for all the elements of the set, the result of the binary operation also belongs to the set;

2) associativity: for all a, b and c in $\mathrm{G},(a \bullet b) \bullet c=a \bullet(b \bullet c)$;

3) identity element: the unique identity element $1_{G}$ exists in group G, such that the equation $a \bullet 1_{G}=1_{G} \bullet a$ holds true for each element $a$ of G;

4) inverse element: for every element $a$ of G, there exists the only inverse element $b$, such that $b \bullet a=a \bullet b=1_{G}$.

If the result of the group operation does not depend on order of group elements, the group is called abelian or commutative.

If the group structure is qualified on a smooth manifold and the group operations of multiplication and inversion are smooth maps, the corresponding group is defined as a Lie group. 
Second, due to the nature of the price and since the impact of transport costs on prices is additive, group action on fiber $F$ is free and transitive ${ }^{6}$. Hence constructed fiber bundle is also a principal bundle ${ }^{7}$.

To sum up, we have built the model of spatially located market as a fiber bundle. This is quite a simple model; nevertheless, it demonstrates advantages of the applied approach over widespread methods to specify transport influence on spatial allocation of economy. It is essential to note that application of theory of fiber bundles to economics is not limited to modeling of market spatial location. Further development of the constructed model and some findings of the presented approach are discussed in section 4. Next section is devoted to formulating the constructed "transport-economy" fiber bundle as a gauge model.

\section{Mathematical model}

The gauge theory is considered the framework of modern field theory in physics. It is based on two main principles: least action and gauge invariance. In our case, the latter means invariance of market equilibrium under spatial reconfiguration of prices.

Next, we will develop a gauge model of the principal bundle constructed in the previous section.

Let denote spatial market allocation by the complex function $\varphi(x)$ :

$$
\varphi(x)=\varphi\left(x_{1}, x_{2}, t\right)=q(x)+i p^{-1}(x),
$$

where $q(x)$ is a local quantity and $p(x)$ is a local price of the commodity. Note: in order to consider market dynamics, $\varphi(x)$ is also defined as a function of time $t$.

Since we model transport costs impact on market allocation, equilibrium is reached through price adjustment. Then we may assume total quantity of the commodity fixed.

To define equilibrium, state the following optimization problem:

$$
\left\{\begin{array}{l}
\min \left|\partial_{i} \varphi(x)\right|^{2} \\
|\varphi(x)|^{2} \leq|\sigma|^{2}
\end{array}\right.
$$

where $\partial_{i} \varphi(x)$ are directional derivatives of (1) at point $x$ of $B$, and $\sigma$ is a complex number (total quantity; inverse minimum price) of the commodity. Inverse minimum price is the inverse of global equilibrium price in the absence of transport costs. We use the product of complex conjugates because here the

\footnotetext{
${ }^{6}$ The action of group $\mathrm{G}$ on the set $\mathrm{X}$ is called free and transitive, if it has the following properties:

1) for different group elements $g$ and $\mathrm{h}$ and any $x \in X$ the following holds true: $g \bullet x \neq h \bullet x$;

2) for any element $x$ of the set $X$, the equation $G \bullet x=X$ holds true.

${ }^{7} \mathrm{~A}$ principal bundle is a fiber bundle (E, $\left.p, B, F\right)$ together with a continuous right group action $\mathrm{E} \times \mathrm{G} \rightarrow \mathrm{E}$ such that $\mathrm{G}$ preserves the fibers $\mathrm{F}$ and acts freely and transitively on them. An alternative definition: it is a fiber bundle, where $\mathrm{p}$ is a smooth mapping between smooth manifolds, $\mathrm{G}$ is a Lie group, and the corresponding group action is smooth.
} 
imaginary unit $i$ is essentially non-distinct from its inverse $-i$. The corresponding Lagrange function is as follows:

$$
L=\partial_{i} \varphi(x) \partial_{i} \bar{\varphi}(x)-\lambda \varphi(x) \bar{\varphi}(x)+\lambda \sigma \bar{\sigma} .
$$

Transition dynamics from one market equilibrium to another is conformed to the principle of least action. The action is defined as a functional of the generalized coordinates $\varphi(x)$ :

$$
S=\int L\left(\partial_{i} \varphi(x), \varphi(x)\right) d^{2} x d t
$$

Then the transportation of the commodity with minimal transport costs is measured as minimum of action $S$. That is, the variation of the action $(\partial S)$ must be zero.

Further, consider transformations of (3), caused by both global and local market reallocations (or changes in trade flows), expressed through changes of $\varphi(x)$.

In the previous section group structure has been added to the set of prices. Values of $\varphi(x)$ are elements of the group as well. Indeed, according to the inequation from the optimization problem (2) values of $\varphi(x)$ vary from zero to $\sigma$. Thus, we may add commutative group structure on the set of values of $\varphi(x)$ in the same way we did it in the previous section for local prices: by defining half the complex number $\sigma$ as the identity element and remeasuring all other elements of the set based on this identity element.

Overall, the group, qualified on $\varphi(x)$, must be the general linear group $G L$ $(n, C)$. However, since we explore transport costs impact on spatial allocation of market, only variations of $\varphi(x)$ caused by transport costs shift are the subject of interest. For our "transport-economy" principal bundle the subgroup of $G L(n$, $C$ ), which acts only on the fiber, is the unitary group of degree 1 , denoted $U(1)$. The action of $U(1)$ corresponds to rotation of the vector $\varphi$ in the complex plane at a given angle around the origin. Thus, it is possible to associate its action with rotation of this vector in the fiber.

Then, spatial reconfiguration (reallocation) of the market is expressed through the multiplication of $\varphi(x)$ on the group element. Since $e^{i \Delta} e^{-i \Delta}=1$, it is obvious that the Lagrangian (3) is invariant under global transformations:

$$
L=\partial_{i}\left(e^{i \Delta} \varphi(x)\right) \partial_{i}\left(e^{-i \Delta} \bar{\varphi}(x)\right)-\lambda\left(e^{i \Delta} \varphi(x)\right)\left(e^{-i \Delta} \bar{\varphi}(x)\right)+\lambda \sigma \bar{\sigma},
$$

where $\Delta$ is a constant (angle of rotation). Global market changes of either price or quantity of the commodity are conformed to the minimum of action and have no impact on the existing equilibrium. However, for local transformations ( $\Delta$ is a function of $x$ ) it does not hold true. Indeed, while $\varphi(x)$ and its complex conjugate change in the same way as in (5), their derivatives do not:

$$
\partial_{i} \varphi(x) \rightarrow \partial_{i}\left(e^{i \Delta(x)} \varphi(x)\right)=e^{i \Delta(x)} \partial_{i} \varphi(x)+\varphi(x) \partial_{i} e^{i \Delta(x)} .
$$

This fact represents that in our model any distortion of allocation of the commodity from the (previous) equilibrium is the endogenous result of local changes in transport costs in the following way. In order to make the expression 
(3) invariant under local transformations, define a new derivative operator such that the derivative of $\varphi(x)$ will again transform identically with $\varphi(x)$ :

$$
D_{i} \varphi(x)=\partial_{i} \varphi(x)+i \rho \tau_{i}(x) \varphi(x),
$$

where $\rho$ is a constant, estimating the strength of influence of transport costs (vector field $\tau(x)$ ) on market allocation. More details on adoption of covariant derivative $D \varphi(x)$ can be found in Appendix, 1 or in (i.e. Makeenko, 2002; p. 85-87). To the covariant derivative (7) transformed correctly, $\tau(x)$ must transform as follows:

$$
\tau_{i}(\mathrm{x}) \rightarrow \tau_{i}(\mathrm{x})-\frac{1}{\rho} \partial_{i} \Delta(x) .
$$

Due to (7) and (8), $\tau(x)$ is equivalent to the components of the connection form of the principal bundle built in the previous section. For detailed explanation see (Moriyasu, 1983; p. 36-40).

It must be noted that introduction of $\tau(x)$ to the Lagrangian results in incompleteness of action $S$. To make it complete again, we must add Lagrangian of $\tau(x)$ to the action (4). Obviously, such Lagrangian must be scalar and invariant under transformations (5) and (6). The simplest one to meet these requirements is the Lagrangian of the trace $\operatorname{Tr}\left(F_{\mu v} F_{\mu v}\right)$ of the curvature form $F_{\mu v}$ :

$$
L_{\tau}=-\frac{1}{4} F_{i j} F_{i j} \text {. }
$$

$F_{\mu \nu}$ describes curvature of a connection (transport costs) on our principal bundle. It is defined by:

$$
F_{i j}=\frac{\partial \tau_{j}(x)}{\partial x_{i}}-\frac{\partial \tau_{i}(x)}{\partial x_{j}} .
$$

To sum up, the complete action, which defines equilibrium dynamics in our model of spatially located market, is as follows:

$$
S=S_{\varphi}+S_{\tau}=\int\left(D_{i} \varphi(x) D_{i} \bar{\varphi}(x)-\lambda \varphi(x) \bar{\varphi}(x)+\lambda \sigma \bar{\sigma}-\frac{1}{4} F_{i j} F_{i j}\right) d^{2} x d t .
$$

Now we can derive equations for the dynamics of $\varphi(x)$ and $\tau(x)$ from (11). As it has already been mentioned, they must correspond to the minimum of transportation: derivatives of action $S$ must be zero. Market dynamics of commodity prices and quantities is given by the following equations:

$$
\begin{aligned}
& D_{i} D_{i} \varphi(x)+\lambda \varphi(x)=0 \\
& D_{i} D_{i} \bar{\varphi}(x)+\lambda \bar{\varphi}(x)=0
\end{aligned} .
$$

The equation for the dynamics of transport costs is as follows:

$$
\partial_{i} F_{i j}=-i \rho\left[\bar{\varphi}(x) D_{j} \varphi(x)-D_{j} \bar{\varphi}(x) \varphi(x)\right] .
$$

In order to fill in details about the derivation of the equations (12) and (13), one may refer to Appendix, 2 and 3 or to (i.e. Rubakov, 2002; p. 75-80).

Although the way we have constructed our gauge model is consistent and natural, it raises a technical problem for solution of equations (12) and (13). 
Indeed, invariance of minimum of action (11) under global and local market spatial reconfiguration (reallocation) means that for the given initial conditions if $\varphi(x)$ and $\tau(x)$ is a solution of equations (12) and (13), then some transformed configuration

$$
\begin{gathered}
\tau_{i}^{\prime}(x) \rightarrow e^{i \Delta(x)} \tau_{i}(x) e^{-i \Delta(x)}-\frac{\partial e^{i \Delta(x)}}{\partial x^{i}} e^{-i \Delta(x)} \\
\varphi^{\prime}(x) \rightarrow e^{i \Delta(x)} \varphi(x)
\end{gathered}
$$

is also a solution of equations (12) and (13). In order to get the only solution, some additional condition must be imposed. For instance, it may be the absence of divergence of transport costs or $\partial_{i} \tau(x)=0$. The choice of such a condition depends on the nature of corresponding problem.

To sum up, the derived equations (12) and (13) are the last key element of the theoretical model of spatially allocated market, which we have been developing for two sections. At the same time, they are the basis for any further detail simulations. Although the latter is not one of the aims of this paper, some of its issues will be discussed in the next section.

\section{Further development}

As it follows from (1) - (13), the mathematical model developed in the previous section is devoid of shortcomings, mentioned in the introduction. It describes influence of spatially variant transport costs on spatial allocation of market endogenously and in a natural way (through prices). Although elimination of serious flaws of NEG models itself is important, application of the approach considered in the paper is not limited only to this. Several issues of further development are being discussed below.

1. The theory of fiber bundles is a profoundly developed mathematical discipline. Its rich and powerful mathematical tools make it possible both to revisit some of the empirical facts and theoretical bottlenecks and to develop new areas of research.

One of the facts to be explained is common spatial patterns of transport networks for different types of transport, geographical conditions and historical evolution. To be exact, it is necessary to find out why such patterns appear in some cases, but do not appear in other. Possible explanation lies in characteristic cohomology classes $^{8}$ of constructed fiber bundles. In general, cohomology describes some fundamental features (topological invariants) of topological spaces: the existence of "holes" in space, "twistedness" of bundles and obstructions of creating globally trivial fiber bundles. Technical features of

\footnotetext{
${ }^{8} \mathrm{~A}$ de Rham cohomology group of a smooth manifold $\mathrm{M}$ is a factor group $\mathrm{Z} / \mathrm{B}$, where $\mathrm{Z}$ - an abelian group of closed differential forms and B - an abelian group of exact differential forms. Characteristic cohomology classes are elements of cohomology groups of base spaces of principal bundles.
} 
transport modes, that are under consideration of corresponding fiber bundles, define such topological invariants of base space. It is the natural way to classify spatial patterns of transport networks and thereby to ground theoretically their differences or similarities based on fundamental features of constructed "transport-economy" fiber bundles.

Examples, that illustrate the essence of this hypothesis, are shown on Figure 3, next page. From the geographic point of view, cases a) and b) have nothing in common. Although their spatial patterns of transport networks are quite similar: a transport route, which connects two transport networks. From the "fiber bundle concept" point of view, the latter is an obvious and natural consequence of similarity in topology of base spaces d) and e). Indeed, American continents for maritime transport and mountains of the Swiss Alps for road transport are considered holes in base spaces of the corresponding fiber bundles and form obstructions of developing unified and evenly distributed transport networks. At the same time the Seine does not influence spatial patterns of Paris metro, as it is shown in the case c), as either metro is built underground or it crosses the river by a bridge. Topology of base space f) differs essentially from those of d) and e), and therefore spatial patterns of Paris metro are not similar to those of cases a) and b).

As far as new areas of research are concerned, one of them is the analysis of structure groups of principal bundles of economy. The existence and the nature of symmetries in economic processes, topological characteristics of phase space (of an economy) in regard to topology of either structure groups or holonomy groups of connections are the subjects to be examined. In author's view, it may reveal some of essentially new aspects of spatial factors impact on sustainability of economic growth.

2. Although at the moment the model of spatially located market as a fiber bundle, developed in this paper, is a theoretical one, empirical studies within the framework should not encounter problems. However, detailed empirical tests, as well as further practical application of the approach and the model, is rather a complex and tedious task for several technical reasons. First, while the model, built in previous sections, is quite simple and thus tractable, extending it from the market to the economy, from one commodity to the variety and from modeling only transport costs impact to considering several transport variables will significantly complicate all the required calculations. Second, according to (1) - (13), all the given data must be spatially located, that means economic statistics needs to be structured geographically. Probably, commercial distribution of geographic information systems itself will either solve or ease the latter problem.

3. As it has already been mentioned, applying of fiber bundle concept is not limited to modeling spatial market location. Further development is modeling of economic allocation in space with regard to transport network topology. Obviously, such a model will be more complicated, then the model of 


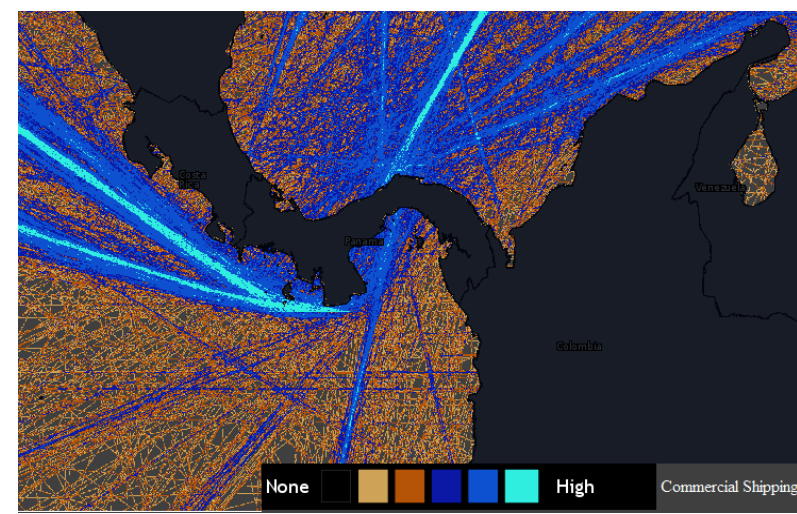

a) Shipping routes through the Panama Canal

Source: NCEAS, UC Santa Barbara

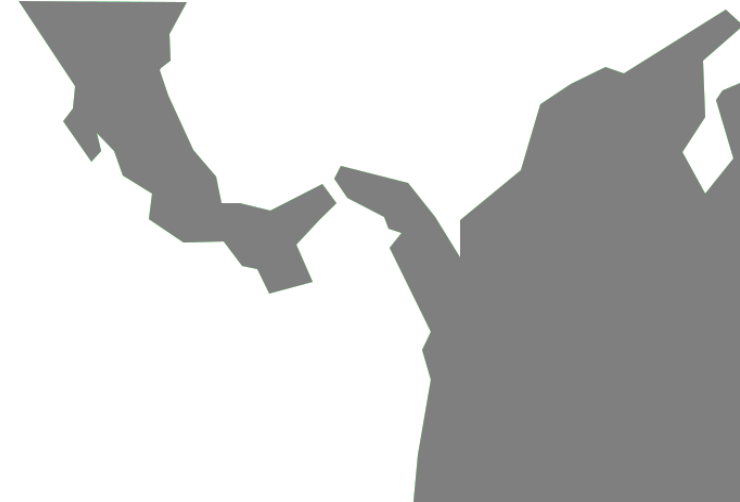

d) base space of "maritime transport" fiber bundle

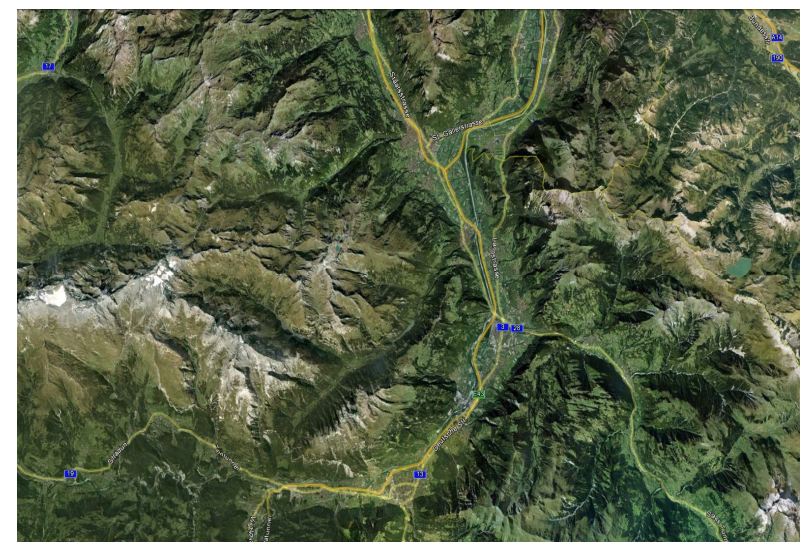

b) Road network along E43 (the Swiss Alps) Source: Google Earth

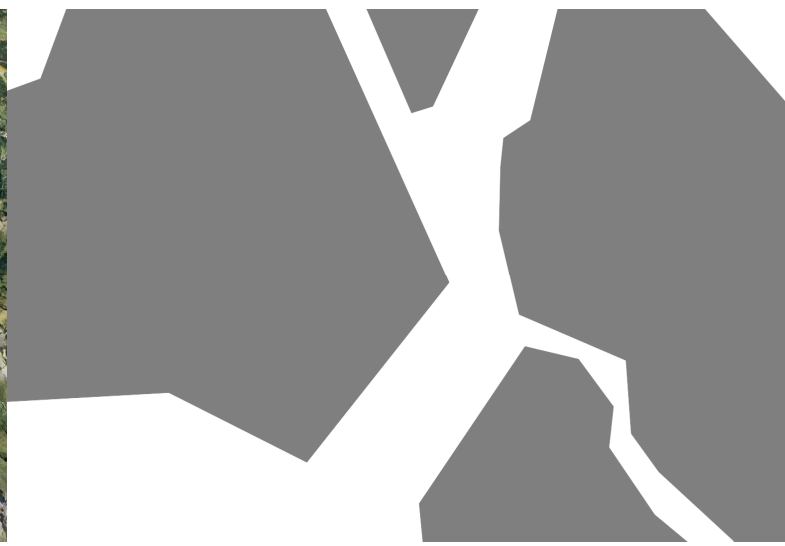

e) base space of "road transport" fiber bundle

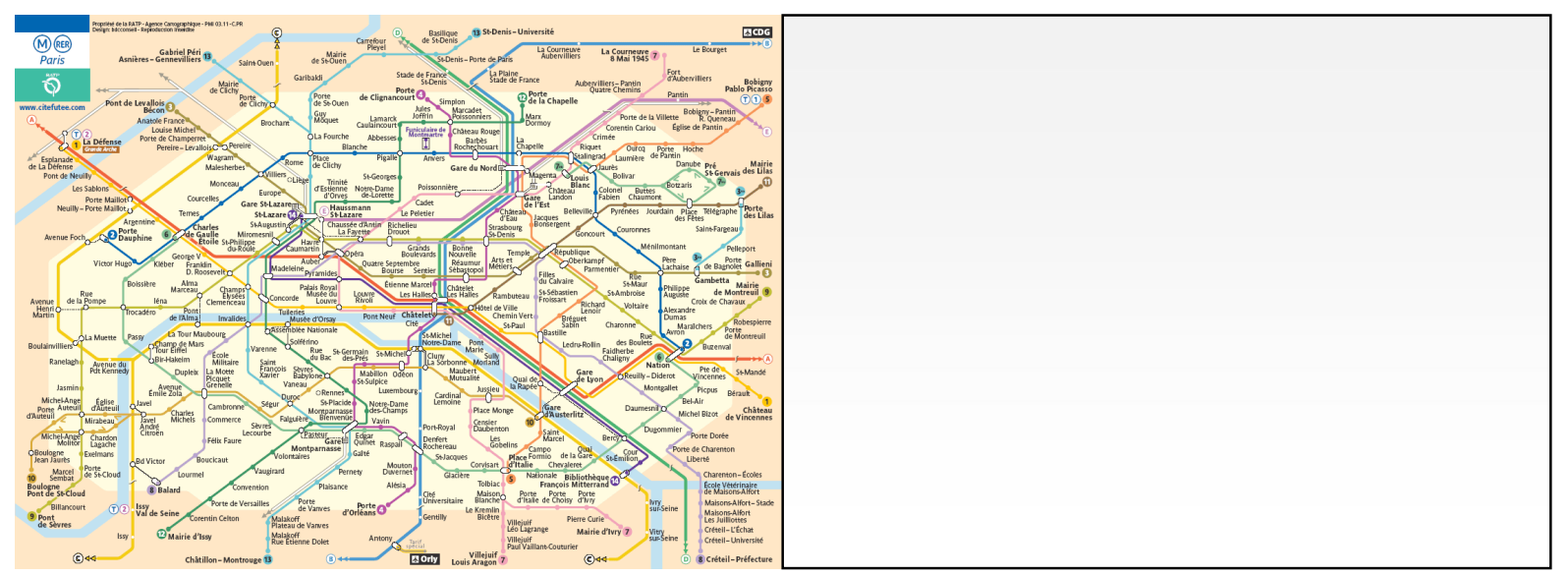

c) Paris Metro Map

f) base space of "metro" fiber bundle

Source: http://mappery.com

Figure 3. Examples of similarities and differences in transport networks and cohomology of base spaces of "transport-economy" fiber bundles

market allocated in space, developed in previous sections. The full action of the corresponding model should consist at least of four parts: the action of economic 
location, the action of trade, the action of transport network location and the action of transportation (vehicle location). That is, it is necessary to detail the research by dividing transport influence on economy into influence of transport network and influence of transportation itself, and by dividing local supply of goods into trade and local production. Such a model would make it possible to simulate long-term evolution and interaction between transport infrastructure and location of economic activities.

Another way to develop the approach is to increase the number of transport variables by adding, for instance, transport density and time of transportation (thus the fiber would have three dimensions instead of one). This is an important and theoretically fruitful task for the next reasons. First, it is the way to enrich theoretical understanding of suppliers' behavior. Indeed, in this case minimization of transportation action by suppliers (due to the principle of least action) would not be limited to minimizing of transport costs; they may as well maximize transport density, minimize time of transportation or combine these variables in an individual manner. Research of simultaneous influence of different transport variables on local varieties of goods is one of the next steps of new economic geography development. Second, it is a must for separation within the approach of transport influence by modes of transport: ship transport, aviation, railway, road transport and so on. Combined with multi-objective suppliers' behavior, mentioned above, this permits to specify and explore the relationship between commodity composition of local varieties of goods and ratio of different modes of transport in transport infrastructure. Third, there are neither empirical no theoretical a priori economic grounds to assume that minimum of transportation action (and thus, equilibrium spatial location of economic activities) will be the same for different transport variables. Hence, if the specification of the gauge model is defined properly, due to the mechanism of symmetry breaking we may model and analyze impact of adoption of new means of transport on division of labor.

Since mathematical tools and notions of the approach, applied in section two, do not depend on the matter of fibration, it is possible to develop other then "transport-economy" fiber bundles. For instance, one of topical tasks for study of modern post-industrial economies is to model and examine impact of innovations in communication on the service sector. We may do this either by developing separate "communication-service" fiber bundle model in the same way as it has been done in section two or by adding relevant variables of communication infrastructure (dimensions to the fiber) to the model of market (or economy) spatial allocation and reconsidering the corresponding notion of traded goods.

To sum up, application of this approach may be extended for analysis of many economic processes, which are under considerable influence of spatial factors. 


\section{Conclusion}

In this paper we presented the approach to specification and modeling of transport influence on spatial allocation of economy. It is essentially new for economic theory in methodology and applied mathematical notions. However, the logic of model construction, used in sections two and three, is quite clear and consistent. Besides, all the given explanations are rather comprehensible than mathematically rigorous. That is why; in some way, this article could be considered an introduction to the presented approach. To examine reasoning in detail, it is necessary to study books and lectures on theory of fiber bundles and gauge theory, to some of which this article refers.

In particular, the model of spatial market allocation with regard to local transport costs has been developed. Its main idea is to "geometrize" some spatial variables by adding an imaginary space (a fiber) for each point of space, in which economy is located, in a locally trivial way (as a product). That is the way to create a fiber bundle. Based on this concept, market, located in space, is defined as a fiber bundle, where local prices have their values in fibers and transport costs are considered the components of the connection form. The crucial advantage of the approach is that endogenous requirement for existence of local transport costs (and transport network) appears, in order to explain distortions of market allocation from the equilibrium (and distortions of trade flows from straight lines).

The existence of local values symmetry of market variables makes it possible to specify such a fiber bundle as a principal bundle and to develop the corresponding gauge model. The latter is based on two main assumptions: the principle of least action and "gauge" invariance. The principle of least action means minimization of transport costs and distance while supplying the commodity. According to the microeconomics, it is sound under assumption of profit-maximizing suppliers. The "gauge" invariance in the developed model is invariance of existence of market equilibrium under its spatial reconfigurations due to price adjustment mechanism. The derived system of differential equations describes market equilibrium dynamics.

It has been shown that the constructed model (and the approach) has several crucial advantages over iceberg form of transport costs, as well as, great potential for further development. Its key features are spatial variance of transport costs, natural form of their influence on market allocation and endogenous interaction between transport costs and local prices.

Finally, in author's view, further development within the framework will be very fruitful. It would lead to revisiting some of unexplained empirical facts and theoretical bottlenecks, as well as, development of new areas of research. For example, similar patterns of transport networks (in spite of different types of transport, landscape and historical evolution) can be explained in a natural way 
by classifying of corresponding "transport-economy" fiber bundles based on cohomology classes of their base spaces. Besides, application of the approach, described in this article, is not limited to modeling and analysis of spatial market location. As well, it may be used for research of other economic phenomena and processes, which are under significant influence of different spatially variant factors. 


\section{References}

1. Aghion, P., Durlauf, S. (2005). Handbook of Economic Growth. North Holland: Elsevier

2. Blaug, M. (1997). Economic Theory in Retrospect. Cambridge University Press.

3. Fujita, M. (1988). A monopolistic competition model of spatial agglomeration: a differentiated product approach. Regional Science and Urban Economics 18, p. 87-124.

4. Fujita, M., Mory, T. (2005a). Frontiers of the New Economic Geography. Papers in Regional Science 84(3), p. 377-405.

5. Fujita, M., Mory, T. (2005b). Transport development and the evolution of economic geography. Portuguese Economic Journal 4(2), p. $129-156$.

6. Husemoller, D. (1993). Fibre Bundles. Springer.

7. Krugman, P.R. (1991). Increasing returns and economic geography. Journal of Political Economy 99(3), p. 483-499.

8. Krugman, P.R. (2011). The New Economic Geography, Now Middleaged. Regional Studies 45(1), p. 1-7.

9. Makeenko, Y. (2002). Methods of Contemporary Gauge Theory. Cambridge University Press.

10. Michor, P.W. (1991). Gauge Theory for Fiber Bundles. Bibliopolis.

11. Mikkelsen, E.I. (2004). New Economic Geography - an introductory survey. Norut Samfunn, Report № SN2004-01.

12. Moriyasu, K. (1983). An elementary primer for gauge theory. World Scientific Publishing Co.

13. Neary, J.P. (2000). Monopolistic Competition and International Trade Theory. University College Dublin and CEPR.

14. Neeb, K-H. (2010). Differential Topology of Fiber Bundles. FAU Erlangen-Nuernberg 2010.

15. Ottaviano, G.I., Thisse, J.Fr. (2000). On economic geography in economic theory: increasing returns and pecuniary externalities. Journal of Economic Geography 1(2) , p. 153-179.

16. Rubakov, V.A. (2002). Classical Theory of Gauge Fields. Princeton University Press.

17. Venables, A.J. (1996). Equilibrium locations of vertically linked industries. International Economic Review 37, p. 341-359.

18. Way, R. (2010). Introduction to connections on principal fibre bundles. Report, University of Surrey. 


\section{Appendix}

1: covariant derivative $D_{i} \varphi(x)$ is invariant under local transformations (spatial reconfiguration of the market, expressed through the multiplication of $\varphi(x)$ on the group element):

$$
D_{i} \varphi(x) \rightarrow D_{i}\left(e^{i \Delta(x)} \varphi(x)\right)=e^{i \Delta(x)} D_{i} \varphi(x)
$$

Proof:

$$
\begin{gathered}
D_{i}\left(e^{i \Delta(x)} \varphi(x)\right)=\partial_{i}\left(e^{i \Delta(x)} \varphi(x)\right)+i \rho\left(\tau_{i}(\mathrm{x})-\frac{1}{\rho} \partial_{i} \Delta(x)\right) e^{i \Delta(x)} \varphi(x)= \\
=\partial_{i} e^{i \Delta(x)} \varphi(x)+e^{i \Delta(x)} \partial_{i} \varphi(x)+i \rho \tau_{i}(\mathrm{x}) e^{i \Delta(x)} \varphi(x)-i \rho \frac{1}{\rho} \partial_{i} \Delta(x) e^{i \Delta(x)} \varphi(x)= \\
=i e^{i \Delta(x)} \partial_{i} \Delta(x) \varphi(x)-i \partial_{i} \Delta(x) e^{i \Delta(x)} \varphi(x)+e^{i \Delta(x)} \partial_{i} \varphi(x)+i \rho \tau_{i}(\mathrm{x}) e^{i \Delta(x)} \varphi(x)= \\
=e^{i \Delta(x)}\left(\partial_{i} \varphi(x)+i \rho \tau_{i}(\mathrm{x}) \varphi(x)\right)=e^{i \Delta(x)} D_{i} \varphi(x)
\end{gathered}
$$

2: derivation of equations for the market dynamics (dynamics of prices and quantities $[\varphi(x)])$ under the principle of least action $(\delta S=0)$ :

$\left.\left.\delta S[\delta \varphi]=\int\left(\partial_{i} \delta \varphi(x)\right)+i \rho \tau_{i}(\mathrm{x}) \delta \varphi(x)\right) D_{i} \bar{\varphi}(x)-\lambda \delta \varphi(x) \bar{\varphi}(x)\right) d^{2} x d t=0$

That is, changes of $\mathrm{S}$ under very small changes of $\varphi(x)$ must be zero.

Let integrate the first term by parts:

$$
\begin{gathered}
\int\left(\partial_{i} \delta \varphi(x)+i \rho \tau_{i}(\mathrm{x}) \delta \varphi(x)\right) D_{i} \bar{\varphi}(x) d^{2} x d t= \\
=\delta \varphi(x) D_{i} \bar{\varphi}(x)-\int \delta \varphi\left(\partial_{i} D_{i} \bar{\varphi}(x) d^{2} x d t-\int \delta \varphi i \rho \tau_{i}(\mathrm{x}) D_{i} \bar{\varphi}(x) d^{2} x d t \approx\right. \\
\approx-\int \delta \varphi\left(\partial_{i}-i \rho \tau_{i}(\mathrm{x})\right) D_{i} \bar{\varphi}(x) d^{2} x d t
\end{gathered}
$$

Then, full change of $\mathrm{S}$ under very small changes of $\varphi(x)$ will be:

$$
\left.\delta S[\delta \varphi]=\int \delta \varphi(x)\left(\partial_{i}-i \rho \tau_{i}(\mathrm{x})\right) D_{i} \bar{\varphi}(x)+\lambda \bar{\varphi}(x)\right) d^{2} x d t=0
$$

This implyies the second equation of (12). In the same way, we may derive the first one (under very small changes of $\bar{\varphi}(x)$ ).

3: derivation of equations for dynamics of transport costs under the principle of least action:

$$
\delta S[\delta \tau]=\int\left(i \rho \delta \tau_{i}(\mathrm{x}) \varphi(x) D_{i} \bar{\varphi}(x)-i \rho \delta \tau_{i}(\mathrm{x}) \bar{\varphi}(x) D_{i} \varphi(x)-\frac{1}{2} F_{i j} \delta F_{i j}\right) d^{2} x d t=0
$$

where

$$
\delta F_{i j}=\frac{\partial \delta \tau_{j}(x)}{\partial x_{i}}-\frac{\partial \delta \tau_{i}(x)}{\partial x_{j}}=2 \partial_{i} \delta \tau_{j}(x)
$$

The latter expression holds true because the first term differs from the second one by substitution $i \leftrightarrow j$ and its sign. Next, let integrate the last term of the former expression by parts: 


$$
\int\left(-\frac{1}{2} F_{i j} 2 \partial_{i} \delta \tau_{j}(x)\right) d^{2} x d t=-\delta \tau_{j}(x) F_{i j}+\int \delta \tau_{j}(x) \partial_{i} F_{i j} d^{2} x d t
$$

Then, the variation of action $\mathrm{S}$ with respect to the variation of transport costs will be:

$$
\delta S[\delta \tau]=\int \delta \tau_{i}(\mathrm{x})\left(i \rho\left[\varphi(x) D_{i} \bar{\varphi}(x)-\bar{\varphi}(x) D_{i} \varphi(x)\right]+\partial_{i} F_{i j}\right) d^{2} x d t=0
$$

This implyies the equation (13). 